\title{
The Unification of Mass and Energy
}

\author{
Edwin Zong \\ Oasis Medical Group Inc., Las Vegas NV USA
}

\section{Email address:}

r4gomed@yahoo.com

\section{To cite this article:}

Edwin Zong. The Unification of Mass and Energy. American Journal of Physics and Applications. Vol. 3, No. 5, 2015 , pp. 178-182. doi: 10.11648/j.ajpa.20150305.13

\begin{abstract}
Our universe is an amazing place where matter floats. The endless stories about changing of mass or energy in the universe may overwhelm all tabloid writers. However, Einstein found a very simple way to cover all the stories in "One" equation: $\mathrm{E}=\mathrm{MC}^{2}$. Yes, the "C" actually stands for speed of light (photon) in the vacuum. Few will argue photons are electromagnetic/radiation power. However, many deny photon's mass status. In this paper, the author will assure you that photon is mass, which also happens to serve the foundation for all gravitational power/positional energy that exists today after a big bang. (1) Furthermore, the author will also assure you that neutrino is the dark matter that floats in the space chilling with electrons $\left(\right.$ and $\left.^{+}\right)$. Again, the author assures you that the famous gamma ray burst is just cosmic-sized of our well-studied experiment in our lab: $\mathrm{e}^{-}+\mathrm{e}^{+} \rightarrow \gamma+\gamma$ ! Our current universe's mass and energy (detectable or undetectable) are all derived from a big bang's photons. The primary objective of this study is to uncover the unified origin for both mass and energy in the universe along with their evolutionary path in the discipline of Newtonian physics.
\end{abstract}

Keywords: Photon, Neutrino, Gamma Ray, Big Bang, Dark Matters

\section{Introduction}

The gravity means that positioned objects are orbiting around another celestial body simultaneously with or without physical contacts. The single orbiting energy/power unifies matter and particles, because particles possess orbiting power/gravity as well when they are coupled.

The object may lose its gravity of significance when it flies out of its orbit. In an enclosed system, without outside influence, a free fall apple may lose its gravity of significance until it touches down the earth then; it regains its gravity of significance. The same principle apparently applies to the photons as well. The un-coupled light photon possesses little positional energy/gravity, but once it is captured and coupled in an apple tree. Such light photon now becomes part of an apple tree or mass with gravity of significance. Being part of an apple, the stationed photons satisfy all definition for mass. The stationed/coupled photons are mass; the separated/single photon is mass as well.

The Particles $(\alpha ! \& \beta)$ and antiparticles $(\alpha \& \beta) \rightarrow$ Gamma rays! Neutrinos etc.

The reverse of "Said Annihilation" $\rightarrow$ Turn Photon $\rightarrow$ Neutrino, $\alpha \& \beta$ particles $\rightarrow$ nucleus! The experiments for matter/antimatter further supports photon's mass status!

The photon bonding/coupling has recently been accomplished in an inorganic lab-a remarkable discovery called Rydberg blockade by coaxing photons into bonding together to form "molecules" - a state of matter by a group led by Harvard Professor of Physics Mikhail Lukin and MIT Professor of Physics Vladan Vuletic. (2) According to the conservation law of mass, the mass of new "molecules" must come from somewhere rather than from nothing, therefore photons have mass.(1) At the beginning of a big bang or spontaneous combustion, the temperature and pressure are fiercely high; few would argue that any other form of mass has a chance to exist other than radiation itself which is a big bang's photon! The author has subsequently linked gamma ray's photons to a big bang. (3)

When a light photon is not paired, it possesses pure kinetic energy/radiation power with little gravitational effect (massless paradox), e.g. a cosmic ray! If it is paired e.g. photon bonding or photosynthesis, it possesses positional energy/gravitation (mass paradox for some people). (3)

When a photon is out of its orbiting status, it carries little gravity, but it doesn't mean that it lost its mass's status. Once again, our nature is never penny pinching to provide us facts for helping us understand its mechanism.

The author has reasoned our universe's dark matter is mainly made of neutrinos! The neutrino background is still present today, with about 56 electron neutrinos, 56 electron 
anti-neutrinos, 56 muon neutrinos, etc., per cubic centimeter, for a total of 337 neutrinos per cubic centimeter in the Universe (45\%). The photons of the CMBR are slightly more numerous with 411 photons/cc (55\%). The Neutrinos is second abundant raw material available in our cosmos which makes it \#1 candidate for our dark matter/black hole!

The light polarization or gravity lensing effects are perfect examples reminding us that photon has mass. The light can be bent, simply because light is mass. The discovery of gravity lensing effects should not be more exciting than your polarized kitchen's light that is bent by your MФNSTER drink.

The photon and neutrino carry no electric charge, but the electron carries positive or negative charge. The only mechanism that can be deduced is "polarization of photon bonding". The neutrino is neutral or "even bonding" of photons. The positive or negative charged electron is polarized or "non-evenly bonding" of photons, which is heavier or massive on one end than the other end!

Our observable universe is spectacular; however, if we are willing to do some detective work, we will find out that all mass with gravitational energy can be traced down to photons. (1) Meanwhile, all electromagnetic power and nuclear force can be traced down to photons as well!

The author, therefore, deduces that there are two forms of fundamental force/energy existing in our universe. 1. Gravitation. 2. Electromagnetic power.

Many people are overwhelmed by standard mode of particles. Following the footprint of Einstein's legend- "One" Equation theory $\left(\mathrm{E}=\mathrm{MC}^{2}\right)$ fits all, the author postulates an incredible "single particle" table for a busy bee- "two" particle mode theory, which are photon and neutrino!

In reality, the radiation and its bigger brothers/sisters e.g. protons, neutron, electrons, atoms are all we need to know in our daily life for engineering any goods. Anything in between is just transient with few practical application. The photons, however, are uniquely important. The author has deduced that photons can be used in optical computing (3) (4) (5) and dark matter engineering along with medicine and defense systems. (3) The photon's research apparently has made a great leap since the beginning of 2015, with laser beam reaches scorching 2 petawatt peak power in the Institute of Laser Engineering at Osaka University, Japan. A petawatt is equal to a quadrillion watts - total energy consumption worldwide in 2012 was estimated by the International Energy Agency (IEA) to be 155.5 petawatt hours (PWh). Ten petawatt lasers are also planned by other institutes in Europe and China, among other places. (6) As its energy approaches the level of a big bang's ray, those super mega-power lasers will become invaluable assets for our replicating the universe's origin and its evolution path in our lab, as well as its practical applications that can be endless. (7)

\section{The Mass, The Energy and The Speed}

The universe maybe big and spectacular, but its message is fairly simple. It is all about the gaining or losing of mass/energy in a vast space/void, generating some "wow" effects (e.g. changing of color or velocity). After removing all juicy details, Einstein saw one thing in the universe: $\mathrm{E}=\mathrm{MC}^{2}$.

The universe is an enclosed system where nothing exists outside. Given the open space within, the little "loss or gain" of mass/energy happens all the time. Subsequently, the changing of mass/energy will lead to the changing of velocity in an enclosed system without outside influence e.g. the universe. Therefore, Einstein's equation tells us, if we artificially increase our mundane velocity to the speed of light in the vacuum (without outside influence); either our own Energy or our mass must change accordingly! Say, if we keep our own mass unchanged, to satisfy Einstein's equation, our energy will be increased enormously till our mass is vaporized into rays! On the other hand, if we keep our own energy unchanged, to satisfy Einstein's equation, our mass will be shredded into extremely tiny size which happens to have another name - rays as well!

The Einstein's equation tells us that there will be no chance for you to flash your lights if your body is to be accelerated to a speed of light in a vacuum, stamping out relativity into Disney world outside of his equation.

The Einstein's equation also tells us, the changing of velocity is secondary to mass/energy's changing in an enclosed system (without outside influence). In the universe's enclosed system, the mass/energy's changing is original and independent; the velocity or "time-scaled" process is the result from such said mass/energy's changing; it is not the other way around! For instance, your dad owns a spectacular house and garden, but its base for his spectacular home is simple-his wage. If there is anything changing in his magnificent house, it will be the result of his income (mass/energy) gain or loss, not the other way around. The "time-scaled" process is equivalent to his spectacular house, the driven force, however, is based on mass/energy's gaining or losing (your dad's wage gaining or losing), therefore, the spectacular "wow" process/time scaled process is the result not the cause!

The introduction of arbitrary time unit is for human's calculation convenience. The artificial time is irrelevant to the changing of the universe's mass/energy.

The Einstein's equation also tells us, there is no place in his equation for space, because the space is just a void.

The Einstein's equation successfully covers all paths of our universe's mass/energy evolution, stamping out "spacetime" to a fabricated land outside of his equation. The Einstein's equation reminds us to think logically based on nature's fact (not imaginary scenery) and principle of "experimental-proved" physics, stamping out all fortunateteller's great prediction.

\subsection{The Photon Myth}

If you happen to stumble into an empty chamber (a total "void" space); before you pass out, will you believe you see your ex's image out of the void? Or feel your ex's energy 
from the void? Unless you believe in magic, it is impossible. However, if you see a light; will you believe the light comes to you with no carrier? Assuming you are sober. Can author safely reason that the photon/light must have a carrier (which is a mass, of course)? If there is anything that can be considered dominating our universe at any moment, it has to be photon! If one accepts photon's mass status, one can immediately realize how simple our universe's mechanism is As John Wheeler once said, "Some principle uniquely right and uniquely simple must, when one knows it, be also so obvious that it is clear that the universe is built, and must be built, in such and such a way and that it could not possibly be otherwise."

It has been estimated that dark matter may make up $84.5 \%$ of the total matter in the universe. (12) The big bang's radiation or high energy photons or gamma ray will penetrate any mass that exists during a big bang explosion while some were polarized. For any gamma ray photons to be trapped or paired at any sub-nucleon level, those storming photons need to be "slowed down" first! Otherwise, the emulsion will not work with storming rays at the speed of light! The missing link is how lights can be slowed down; now the puzzle is finally solved! At a couple degrees above absolute temperature zero, the lights appear "slowed down" significantly where ubiquitous emulsion stands for a chance to work! (2) Our universe's background temperature is $2.7 \mathrm{~K}$. It might go further down when our region of universe is ripe for a next big bang; however our background temperature will never reach absolute temperature zero. (3)

The big bang Nucleosynthesis starts with merging /coupling of big bang's high energy particles/photons in the soup of electrons and Cosmic Microwave (initial big bang's kinetic energy along with emulsion force) $\rightarrow$ protons and neutrons/primordial nucleons formation (some big bang's kinetic energy transfer to positional or orbital energy also known as gravity) $\rightarrow$ the "initial" or unstable nucleus's decay/radioactive decay $\rightarrow$ dark matter/neutrino congregation/ stabilization $+\lambda$ ray bursts $\rightarrow$ mass gathering due to dark mater's gravity $\rightarrow$ visible galaxy formation.

The dark matter/neutrino congregation may go thru "initial formation" first, then "stabilization" with radioactive decay: (Cownan-Reines neutrino experiment) beta negative decay reacted with protons to produce neutrons and positrons which quickly combine with electrons, releasing gamma rays. (13)

The significant part of mass after a big bang apparently is stuck in the most basic form of mass-dark matter, the paired/bundled photons chilling with their mate-electrons. The halo of rich electric activities (e.g. x-ray), therefore, will be expected from any dark matter of substantial size! The author reasonably expects more activities happen among electrons within or surround dark matters, which may strike and trigger "dark matter signature" $\mathrm{X}$ ray. The author's view is supported by X-ray emission observed from the center of galaxies. (14)

The cosmic background wave and space radiation make up the rest (the majority part of our universe's mass) of "dark matter" since most scientists turn their eyes away from photons' mass status. The cosmic rays/radiation should not be called "dark" mass, but for now, may I name them "illegitimate children" or "by-blows"?

Without any fancy language, the path of particle in the universe is fairly simple: Radiation/photon $\rightarrow$ dark matter/neutrino congregation $\rightarrow$ visible matter/inorganic matter $\rightarrow$ organic matter $\rightarrow$ you $\rightarrow$ collapse $\rightarrow$ pre big bang dark mass /neutrino congregation $\rightarrow$ big bang $\rightarrow \rightarrow \rightarrow$ you again!

\subsection{The Universe Inflation and Focal Collapse}

There is another space's evidence supporting our big bang theory. It is swirl-like/light polarization imprint on light less than one-trillionth of a second old from our big bang. (8) Such light polarization becomes possible when a big bang's light encounters mass existing during a big bang. The photons of big bang or spontaneous combustion encounter pre-existing masses which support the author's one universe, endless cycle theory. (7) If our space were a void, the light's polarization simply will not happen. The author's model of universe-"one universe endless cycles" is also supported by discovery of Methuselah star, HD 140283 which is "older" than our current universe. HD 140283 appeared to be 16 billion years old, more than two billion years older than the rest of the cosmos (measured to be $13.78 \pm 0.037$ billion years old)! (9) It is simply not paradox; it is another evidence supports universe's endless cycles in a realm of gravity and emulsion. (13)

The evolution of a big bang simply means that some of its greatest kinetic energy starts to be captured into its form of orbital energy/gravity during its cooling off stage, when galaxies start to be created. The destiny of a big bang is another big bang! The gravity along with power of emulsion (another form of gravitation) will eventually attract enough mass which will collapse into an ever increasingly big new pre-big bang dark matter!

The more mass is collected, the bigger a pre-big bang dark matter grows, which manifests stronger gravity-directly evidenced by our observation of accelerated universe's expanding! The accelerated speed is fueled by growing mass/increasing gravity power!

Our observed universe has passed a new born stage of post big bang, passed an adult stage of "galaxies' manufacturing boom". Our observed universe is entering a big bang or spontaneous combustion's preparing stage-senior age (our observed universe is getting old!), which is evidenced by fewer new galaxies' forming. The accelerated expanding is another direct evidence of stronger gravity pull - larger and larger "pre-big bang" dark mass/neutrino congregation is forming! For those who use accelerating universe as a reason doubting the big bang theory, the author can assure those doubters that the opposite is true!

Furthermore, our galaxies are inflating because of star's photons. Without radiations from stars, our galaxies' mass may collapse into dark matter which is indirectly supported by supernova effects. (10)

The initial universe's inflation is powered by a big bang's 
photons; the secondary inflation which occurs in galaxies is fueled by their star's photons. The focal collapse is due to the fact that their stars run out of fuels, which subsequently age galaxies to their dwarf's stage/senior citizen, which are mostly made of dark matter along with fewer stars. It is evidenced by discovering dwarf galaxies in our current universe. (11)

Our universe is simply trillion and trillion light years big (infinite!), there are numerous focal collapses which are preparing for a next big bang. 14 billion light years is just a tiny drop of water in an infinitely gigantic size of our universe. Don't trip, it is just like that!

\subsection{The Energy Evolution in the Universe}

The universe is a complete and enclosed system, because there is nothing existing outside our universe which may interfere with our universe's matter/energy. The conservation law of mass and energy perfectly apply universe as a unit. We can safely say that universe's energy and mass are constant.

Ebigbang $=\mathrm{E}_{\mathrm{k}}+\mathrm{E}_{\mathrm{p}}$

The $E_{k}$ is the sum of kinetic energy presents in the current universe. $E_{p}$ is the sum of positional energy present in the current universe. (3)

When a light/photon is not coupled, it possesses pure kinetic energy/radiation power with little gravitational effect. If it is coupled e.g. photon bonding or photosynthesis, it possesses positional energy/gravitation. (3) The destiny of a single photon/ray is to "marry" another photon/ray; the destiny of "married" photons/rays is to be separated from its partner. The endless cycle of "marry" and "separation" of particles is closely mirroring the endless cycle of energy. The destiny of kinetic energy is to be transformed into positional energy; the destiny of positional energy is to be transformed into kinetic energy.

The conservation law of energy dictates that all nuclear power (strong or weak) existing after a big bang derives from electromagnetic power which is originated from a big bang's radiation-photons as well.

Instead of 4 basic forces, the author promotes 2 fundamental forces in the universe. 1. Gravitational/orbiting force. 2. Electromagnetic/radiation forces. Both are originated from "One" source-photon! Don't trip, it is just like that!

\subsection{The Role of Bonding in Physics and Spirit/Mind}

The Organic Matters branch off from inorganic matters. The process requires light energy. The light energy could come from solar or earth itself. The most popular photosynthesis/photon bonding requires green chlorophyll pigments. In plants, these proteins are held inside organelles called chloroplasts, which are most abundant in leaf cells, while in bacteria they are embedded in the plasma membrane.

The first photosynthetic organisms probably evolved early in the evolutionary history of life and most likely used reducing agents such as hydrogen or hydrogen sulfide as sources of electrons, rather than water. (15) Cyanobacteria appeared later, and the excess oxygen they produced contributed to the oxygen catastrophe, (16)which rendered the evolution of complex life possible.

The Organic Matters are mass possessing both kinetic energy and positional energy. To become alive and grow, the living beings require additional matters that possess almost pure forms of kinetic energy -light energy. However, nothing will stay alive without bonding process.

The bonding is the key for matter's evolution. The bonding is therefore a key factor for human's life. Other than physics, our human mind needs bonding factor too. Such bonding factor is simply known as a focus factor in human $\mathrm{mind} /$ spirit. Such focus factor can be a real stuff or a Phony stuff. In our mind or spiritual life, both will do the trick to provide a focus preventing our mind's scattering. The "Bonding in physics" provides us a scientific ground to understand how beliefs work in human mind and all religions/beliefs are equal. It is just like that!

\section{Results Analysis}

At the beginning of a big bang, the temperature and pressure are fiercely high; few would argue that any other form of matter may stand a chance to exist other than radiation itself which is a photon. The author has subsequently linked gamma ray's photons to a big bang. (3) Furthermore, the author will also assure you that neutrino is the dark matter that floats in the space chilling with electrons $\left({ }^{-}\right.$and $\left.{ }^{+}\right)$. Again, the author assures you that the famous gamma ray burst is just cosmic-sized of our well-studied experiment in our lab: $\mathrm{e}^{-}+\mathrm{e}^{+} \rightarrow \gamma+\gamma$ !

At a couple degrees above absolute temperature zero, the lights appear "slowed down" significantly where ubiquitous emulsion stands for a chance to work! (2) The most primitive mass/dark matter starts to form thru photon bonding. (1) Without any fancy language, the path of particle universe is deduced as follows: Radiation/photon $\rightarrow$ dark matter/neutrino congregation $\rightarrow$ visible matter/inorganic matter $\rightarrow$ organic matter $\rightarrow$ you $\rightarrow$ collapse $\rightarrow$ pre-big bang dark mass/neutrino congregation $\rightarrow$ big bang $\rightarrow \rightarrow \rightarrow$ you again!

Following the path of Einstein's legend of "One" Equation for all, instead of standard model of particle; the author promotes " 2 " particle table which are photon and Neutrino!

The universe is a complete and enclosed system, because there is nothing existing outside the universe which may interfere with the universe's matter/energy. The conservation law of mass and energy perfectly apply universe as a unit. We can safely say that universe's energy and mass are constant.

$$
\mathrm{E}_{\text {bigbang }}=\mathrm{E}_{\mathrm{k}}+\mathrm{E}_{\mathrm{p}}
$$

The $E_{k}$ is the sum of kinetic energy presents in the current universe. $E_{p}$ is the sum of positional energy present in the current universe. (3)

The conservation law of energy dictates that all nuclear power (strong or weak) existing after a big bang derives from electromagnetic power which is originated from a big bang's 
radiation-photons.

Instead of 4 basic forces, the author simplifies it for 2 fundamental forces for practical application. 1. Gravitation/orbiting energy. 2. Electromagnetic/radiation forces. Both are originated from "one"- photon!

\section{Discussion}

The neutrino background is still present today, with about 56 electron neutrinos, 56 electron anti-neutrinos, 56 muon neutrinos, etc., per cubic centimeter, for a total of 337 neutrinos per cubic centimeter in the Universe $(45 \%)$. The photons of the CMBR are slightly more numerous with 411 photons/cc $(55 \%)$. The Neutrinos is second most abundant raw material available in our cosmos which makes it \#1 candidate for our dark matter/black hole!

The conservation law of mass and energy perfectly apply our universe as a unit. Therefore, the author reasons: 1 . The conservation law of mass dictates that a photon is the "single" unified origin for all mass. 2. The conservation law of energy dictates that a photon energy/radiation is the "single" unified origin for all electromagnetic power (includes nuclear forces) as well as all gravitational power/positional energy where photon's mass serves the "single" unified base for all of our universe's mass (detectable or undetectable). The conservation law of mass and energy perfectly supports the "theory" of Einstein's "One" Equation for all!

If we were to set aside our urge for sophistication and fantasy for genie, following the path of Einstein's legend in "One" Equation theory $\left(\mathrm{E}=\mathrm{MC}^{2}\right)$, you will see that a photon is a "single" unified worship for all branches of sciences along with philosophy and religion. The universe is just another glorified "two in one package"-space (void) and photons. Our dark matter/black is just made from Neutrinos or coupled/bundled photons!

The photon and neutrino carry no electric charge, but the electron carries positive or negative charge. The only mechanism that can be deduced is "polarization of photon bonding". The neutrino is neutral or "even bonding" of photons. The positive or negative charged electron is polarized or "non-evenly bonding" of photons, which is heavier or massive on one end than the other end!

The author promotes photon research for its practical application in future computer, medicine, and military along with the universe's mechanical study.

\section{References}

[1] Edwin Zong, Dark Matter Engineering, American Journal of Physics and Applications. Vol. 3, No. 3, 2015, pp. 106-111. doi: 10.11648/j.ajpa.20150303.17
[2] M. Lukin Harvard and V. Vuletic MIT Nature Sep 25, 2013.

[3] Edwin Zong. The Real Universe II. American Journal of Physics and Applications. Vol. 3, No. 3, 2015, pp. 77-85. doi: 10.11648/j.ajpa.20150303.13

[4] McAulay, Alastair D. (1991). Optical Computer Architectures: The Application of Optical Concepts to Next Generation Computers. New York, NY: John Wiley \& Sons. ISBN 0-47163242-2.

[5] Mortaza Noshad, Amin Abbasi, Reza Ranjbar, Reza Kheradmand.(2012) Novel All-Optical Logic Gates Based on Photonic Crystal Structure. Journal of Physics: Conference Series350 (2012) 012007

[6] Eric Mack 2015 "Death Star" laser sets new world record in Japan Science

[7] Edwin Zong. 2014 One Universe, Endless Cycles. Science Research. Vol. 2, No. 5, 2014, pp. 105-110. doi: 10.11648/j. sr.20140205.15

[8] Gary Robbins 2014 Scientists see 'fingerprint' of Big Bang The San Diego Union-Tribune

[9] A. J. Gallagher et al. (2010). "The barium isotopic mixture for the metal-poor subgiant star HD 140283". Astronomy and Astrophysics 523: A24. arXiv:1008.3541. Bibcode:2010A\&A...523A..24G. doi:10.1051/0004$6361 / 201014970$

[10] Ott, C. D. et al. (2012). "Core-Collapse Supernovae, Neutrinos, and Gravitational Waves". Nuclear Physics B: Proceedings Supplement 235: 381. arXiv:1212.4250. Bibcode:2013NuPhS.235..381O. doi:10.1016/j.nuclphysbps.2013.04.036

[11] Josh Simon (2005). "Dark Matter in Dwarf Galaxies: Observational Tests of the Cold Dark Matter Paradigm on Small Scales"

[12] Sean Carroll, Ph.D., Cal Tech, 2007, The Teaching Company, Dark Matter, Dark Energy: The Dark Side of the Universe, Guidebook Part 2 page 46, Accessed Oct. 7, 2013.

[13] C. L Cowan Jr., F. Reines, F. B. Harrison, H. W. Kruse, A. D McGuire (July 20, 1956). "Detection of the Free Neutrino: a Confirmation". Science 124 (3212): 103-4. Bibcode: 1956Sci...124..103C. doi:10.1126/science.124.3212.103. PMID 17796274.

[14] Tauris \& van den Heuvel (2006), "Formation and evolution of compact stellar X-ray sources", In: Compact stellar X-ray sources. Edited by Walter Lewin \& Michiel van der Klis. Cambridge Astrophysics Series, p.623-665, DOI: $10.2277 / 0521826594$.

[15] Olson JM (May 2006). "Photosynthesis in the Archean era". Photosyn. Res. 88 (2): 109-17. doi:10.1007/s11120-0069040-5. PMID 16453059

[16] Buick R (August 2008). "When did oxygenic photosynthesis evolve?". Philos. Trans. R. Soc. Lond., B, Biol. Sci. 363 (1504): 2731-43. doi:10.1098/rstb.2008.0041. PMC 2606769. PMID 18468984. 